\title{
Sperm Defect Severity Rather Than Sperm Source Is Associated With Lower Fertilization Rates after Intracytoplasmic Sperm Injection
}

\author{
Sidney Verza Jr, Sandro C. Esteves \\ Androfert, Center for Male Reproduction, Campinas, Sao Paulo, Brazil
}

\begin{abstract}
Objective: To evaluate the impact of sperm defect severity and the type of azoospermia on the outcomes of intracytoplasmic sperm injection (ICSI).

Materials and Methods: This study included 313 ICSI cycles that were divided into two major groups according to the source of spermatozoa used for ICSI: 1) Ejaculated (group $1 ; n=220$ ) and 2) Testicular/Epididymal (group 2; n =93). Group 1 was subdivided into four subgroups according to the results of the semen analysis: 1 ) single defect (oligo-[O] or astheno[A] or teratozoospermia-[T], $n=41$ ), 2) double defect (a combination of two single defects, $n=45$ ), 3) triple defect (OAT, $n$ $=48$ ), and 4 ) control (no sperm defects; $\mathrm{n}=86$ ). Group 2 was subdivided according to the type of azoospermia: 1 ) obstructive $(\mathrm{OA}: \mathrm{n}=39)$ and 2$)$ non-obstructive (NOA: $\mathrm{n}=54)$. Fertilization $(2 \mathrm{PN})$, cleavage, embryo quality, clinical pregnancy and miscarriage rates were statistically compared using one-way ANOVA and Chi-square analyses.

Results: Significantly lower fertilization rates were obtained when either ejaculated sperm with triple defect or testicular sperm from NOA patients (63.4 $\pm 25.9 \%$ and $52.2 \pm 29.3 \%$, respectively) were used for ICSI as compared to other groups $(\sim 73 \%$; $\mathrm{P}<0.05)$. Epididymal and testicular spermatozoa from OA patients fertilized as well as normal or mild/moderate deficient ejaculated sperm. Cleavage, embryo quality, pregnancy and miscarriage rates did not differ statistically between ejaculated and obstructive azoospermia groups. However, fertilization, cleavage and pregnancy rates were significantly lower for NOA patients.

Conclusion: Lower fertilization rates are achieved when ICSI is performed with sperm from men with oligoasthenoteratozoospermic and non-obstructive azoospermic, and embryo development and pregnancy rates are significantly lower when testicular spermatozoa from NOA men are used.
\end{abstract}

Key words: spermatozoa; intracytoplasmic sperm injection; azoospermia; oligozoospermia Int Braz J Urol. 2008; 34: 49-56

\section{INTRODUCTION}

Intracytoplasmic sperm injection (ICSI) has been the standard for the treatment of severe male factor infertility. Before ICSI, sperm from men with severely defective spermatogenesis, such those with oligoasthenoteratozoospermia, and sperm retrieved from the epididymis or the testicles, were unable to fertilize the egg - even through conventional in vitro fertilization. When microinjected into the egg using ICSI, however, these gametes have shown the capability of diluting their genetic material, fertilizing and 
producing normal and viable pre-embryos (1-8). Although the use of surgically retrieved or ejaculated sperm from men with severely impaired spermatogenesis for ICSI has greatly improved treatment of severe male infertility, the consequences of using such gametes are not fully known (7).

Many studies have shown conflicting results when ICSI is performed with sperm from different sources (2-8). It is difficult to interpret these results because, apart from few studies, only the sperm source is analyzed and there is no systematic distinction between obstructive and non-obstructive azoospermia. This prevents consideration of the influence of spermatic defects. For instance, an ejaculated semen sample can present varying degrees of sperm abnormalities from absence to severe alterations in all spermatic parameters, as in cases of oligoasthenoteratozoospermia. Spermatozoa from normal, mild/moderate or severe abnormal semen may show different fertilizing abilities after ICSI even though their source is the same, i.e., the ejaculate (8). Also, important physiologic differences are observed in obstructive and non-obstructive azoospermia. While obstructive azoospermic men have normal sperm production, non-obstructive ones have defective spermatogenesis and very limited amount of sperm production, if any, and they may carry several sperm defects, including genetic ones (9). Therefore, it is reasonable to speculate that ICSI results depend not only on sperm source but also on the severity of sperm defect.

The objective of this study was to evaluate the impact of sperm defect severity and the type of azoospermia on the outcomes of intracytoplasmic sperm injection (ICSI).

\section{MATERIALS AND METHODS}

This is a retrospective study including 313 ICSI cycles performed from January 2002 to November 2003. To allow data collection, institutional review board approval was obtained as well as patient written consent. Treatment cycles were divided into two main groups according to the sperm source for ICSI: 1) ejaculated (group 1; $\mathrm{n}=220$ ), and 2) sperm obtained from testicles/epididymis (group 2; $\mathrm{n}=93$ ). Group 1 was subdivided into 4 subgroups according to the anomaly observed in the seminal analyses, which were performed in accordance with the World Health Organization Manual (10), and sperm morphology using Tygerberg's strict criteria (11), as follows: (a) single sperm defect (oligozoospermia - [O]: sperm concentration $<20 \times 10^{6} / \mathrm{mL}$; or asthenozoospermia - [A]: progressive motility < $50 \%$; or teratozoospermia [T]: sperm morphology < $8 \% ; \mathrm{n}=41$ ); (b) double sperm defect, i.e., a combination of two defects described above, $n=45$ ); (c) triple sperm defect (all defects combined: oligoasthenoteratozoospermia [OAT], $\mathrm{n}=48$ ); and (d) control (no sperm defect, $\mathrm{n}=86$ ). The limit of $8 \%$ of spermatic morphology was used since recent studies (12-15) have shown a tendency to reduce the now used normality reference of $14 \%$. Group 2 was subdivided in accordance with the type of azoospermia: 1 ) obstructive (AO: $\mathrm{n}=39$ [epididymis $\mathrm{n}=31$; testicles $\mathrm{n}=8$ ), and 2 ) non-obstructive (ANO: testicles $n=54$ ).

Ovarian stimulation and follicular aspiration - Pituitary suppression was achieved by using intranasal gonadotrophin hormone analog (nafarelin acetate, Synarel, Zodiac) followed by ovarian stimulation with daily doses of 150-300 IU of human menopausal gonadotrophin (HMG or HP-HMG; Ferring). Human chorionic gonadotrophin (hCG; Choragon, Ferring) was used when 2 or more ovarian follicles presented a mean diameter of $18 \mathrm{~mm}$. Thirty-four to thirty-six hours after the hCG administration, an ultrasound-guided transvaginal follicular aspiration was performed under general anesthesia administered intravenously.

Oocyte handling and classification - After follicular aspiration, the tubes with the follicular fluid were transferred to the in vitro fertilization laboratory and examined on stereomicroscopy to identify the corona-cumulus-oocyte complexes (CCOC). Immediately, CCOC chemical treatment with $40 \mathrm{IU} /$ $\mathrm{mL}$ hyaluronidase (Hyase, Vitrolife, Sweden) was performed for 30 seconds. The oocytes were then transferred to microdroplets of culture media (IVF, Vitrolife, Sweden) covered with mineral oil (Ovoil, Vitrolife, Sweden) and kept in an incubator for 1-2 hours at $37^{\circ} \mathrm{C}$ in a $5.5 \% \mathrm{CO}_{2}$ atmosphere. Mechani- 
cal oocyte denudation was then performed with a $130 \mu \mathrm{m}$ diameter pipette (Flexipet, Cook, USA) and the denuded oocytes were classified according to their maturity into metaphase II (MII, oocytes showing the extrusion of the 1st polar corpuscle), prophase I (oocytes at the germinative vesicle stage), metaphase I (oocytes showing no germinative vesicule or extrusion of the 1st polar corpuscle), atresic (oocytes with signs of degeneration) and fractured (oocytes with rupture in the zona pelucida with total or partial extrusion of cytoplasm).

Sperm retrieval in group 1 - Sperm was obtained by ejaculation after 48-72 hours of sexual abstinence, and the sample was kept at $37^{\circ} \mathrm{C}$ for 30 minutes or until complete liquefaction. Sperm washing was performed by using the two-layer discontinuous colloidal gradient (Isolate, Irvine Scientific, USA). The ejaculate and the gradients were centrifuged for 25 minutes at $300 \mathrm{Xg}$. The supernatant was discharged and the bottom layer was subsequently diluted with HEPES-buffered culture medium (modified HTF, Irvine Scientific, USA; 1:2, v/v), and washed again by centrifugation for 10 minutes. The pellet was re-suspended in 200 microliters of a HEPESbuffered culture media and maintained at $37^{\circ} \mathrm{C}$ until the time of ICSI. An aliquot was removed for the assessment of sperm count and motility.

Sperm retrieval in group 2 - Sperm retrieval from the testis or epididymis was performed under intravenous anesthesia with propofol in association with blockage of the spermatic cord with $2 \%$ xylocaine on an outpatient basis. Sperm retrieval from the testis was done percutaneously by testicular sperm aspiration (TESA) using a 21-gauge needle or by microsurgical-guided open biopsy (testicular microdissection, micro-TESE) (16). The seminiferous tubules were dissected mechanically under stereomicroscopy in HEPES-buffered culture medium (Modified HTF, Irvine Scientific, USA). The supernatant was collected for sperm search. Sperm retrieval from the epididymis was performed by percutaneous epididymal sperm aspiration (PESA) using a 13.5 gauge needle connected to a $1 \mathrm{~mL}$ syringe. Aspirates were diluted in HEPES-buffered culture medium. In all cases of TESA, micro-TESE or PESA, the samples were centrifuged at $300 \mathrm{Xg}$ for 10 minutes. The pellets were subsequently resuspended in HEPES-buffered culture medium and kept at $37^{\circ} \mathrm{C}$ until microinjection. Sperm search was performed at $400 \mathrm{X}$ magnification using a phase-contrast inverted microscope. In the non-obstructive azoospermia cases, sperm retrieval was performed by TESA or micro-TESE, whereas PESA was used to obtain sperm in obstructive azoospermia cases. However, in 8 cases of obstructive azoospermia, PESA was not successful, and the TESA technique was used to obtain sperm for ICSI in these cases.

Gamete micromanipulation and microinjection - Microinjections were performed at X400 magnification on a $37^{\circ} \mathrm{C}$ heated stage phase-contrast inverted microscope (Nikon, Japan) (1). A Petri dish containing a $4 \mu \mathrm{L}$ microdroplet of PVP (polyvinilpirrolidone, Vitrolife, Sweden) under mineral oil was used for sperm selection and immobilization. On the same dish, a $20 \mu \mathrm{L}$ microdroplet of culture medium (Gamete, Vitrolife, Sweden) was used for placing the oocytes for microinjection. A single sperm was mechanically immobilized by using the tip of the microinjection needle (Cook, USA) and then aspirated inside the needle. The oocyte was held in place using a 35 degree angle holding micropipette (Cook, USA) with the polar body in the 6 or 7 o'clock position. Injection of a single spermatozoon within the oocyte cytoplasm was performed by using electrohydraulic micromanipulators (Narishige, Japan). After all microinjections from a single case were completed, the injected oocytes were transferred to a closed culture system (microdroplets under mineral oil, series II, Vitrolife, Sweden), and incubated for $16-18$ hours at $37^{\circ} \mathrm{C}$ and $5.5 \% \mathrm{CO}_{2}$ until fertilization was determined.

Fertilization and cleavage verification - Fertilization was considered normal when two pronuclei $(2 \mathrm{PN})$ were visualized and the extrusion of the 2nd polar corpuscle was observed. The presence of only one or 2 or more pronuclei was considered abnormal fertilization. After zygote formation, pre- embryo cleavage was verified after 48 hours (day 2) and 72 hours (day 3). Pre-embryos were classified as good quality when they exhibited 3 to 4 symmetric blastomeres on the second day of culture and 7 to 8 symmetric blastomeres on the third day of culture, with absence of 
multinucleation, grades I (absence of fragmentation) or II (up to $20 \%$ of vitelineous space occupied by fragments) of cytoplasmic fragmentation, and absence of zona pelucida alterations (17).

Embryo transfer - All embryo transfers were performed around 72 hours after ICSI. A delicate two-step catheter (Sidney IVF, Cook, USA) was used to place the embryos inside the uterine cavity. Transvaginal embryo transfers were guided by abdominal ultrasound.

Main outcome measures - Descriptive parameters such as female age and mean number of oocytes retrieved were compared among subgroups. After ICSI, the following parameters were analyzed and compared: normal (2PN) and abnormal fertilization rates, cleavage and good quality pre-embryo rates on days 2 and 3, number of pre-embryos transferred, clinical pregnancy and miscarriage rates. A clinical pregnancy was confirmed by the presence of a gestational sac with an embryo exhibiting cardiac activity on a 5th to 6th week ultrasound scan. Miscarriage was considered when a non-viable clinical pregnancy was noted on ultrasound follow-up.

The Kolmogorov-Smirnov test was used to verify if data followed normal distribution. A one-way ANOVA or Fisher exact test was used to compare clinical and laboratory parameters between groups when appropriate. The Chi-square test was used to compare pregnancy and miscarriage rates, with $\mathrm{P}<$ 0.05 considered significant (18). Statistical analysis was performed by StatSoft program, Tulsa, EUA.

\section{RESULTS}

Female age, number of mature oocytes retrieved and number of pre-embryos transferred were not statistically different among groups (Table-1).

Significantly lower normal fertilization rates were obtained when ejaculated sperm with triple sperm defect $(63.4 \pm 25.9 \%)$ or testicular sperm from patients with non-obstructive azoospermia (52.2 \pm $29.3 \%$ ) were used for ICSI in comparison with other groups $(71.3-73.6 \%)(\mathrm{p}<0.05$, Table-2).

There was no difference in fertilization rates when sperm from the epididymis $(74.7 \% \pm 21.2 \%)$ or the testicles $(69.1 \% \pm 19.6 \%)$ of patients with obstructive azoospermia was used for ICSI, as compared with ejaculated sperm with mild (single sperm defect; 73.2 $\pm 22.1 \%$ ) to moderate (double sperm defect; $72.1 \pm$ $19.6 \%$ ) alterations (Tables-2 and 3). Cleavage, preembryo quality, clinical pregnancy and miscarriage rates were not statistically different between ejaculated and obstructive azoospermia (OA) groups, independent of whether the spermatozoa from obstructive azoospermic patients was obtained from the epididymis or testicles (Table-3). However, cleavage rates, pre-embryo quality, and clinical pregnancy were significantly lower in non-obstructive azoospermia (NOA) group, when compared to the other groups (Table-2).

\section{COMMENTS}

Several studies report ICSI results based on the sperm source rather than on sperm defect severity. Sperm source criteria, however, may include spermatozoa from different etiologies. For instance, ejaculated semen may contain slightly abnormal spermatozoa from a man with a moderate varicocele, but it may also harbor severely defective sperm from men with genetic disorders, such as the Klinefelter syndrome and AZFc Y chromosome microdeletions. Furthermore, in cases of azoospermia, sperm from the epididymis and the testicles can be used for ICSI. However, spermatogenesis in men with obstructive and non-obstructive azoospermia is very distinct. While sperm production is normal in the former, it is severely abnormal, if existing, in the latter, despite the fact that in both cases ICSI may be performed using testicular spermatozoa (16).

Studies comparing the results of ICSI based on sperm source are conflicting. Some studies have reported similar fertilization and pregnancy rates using ejaculated or epididymal spermatozoa $(2,19,20)$, and both perform better than testicular sperm in terms of normal fertilization rates, embryo quality and/or pregnancy rates (4,5,7,19-21). Other studies report impaired fertilization rates with epididymal spermatozoa but similar pregnancy rates (3), and others observed similar fertilization rates but impaired pregnancy rates (6). However, most of them fail to analyze ICSI results in relation to the se- 
Table 1 - Descriptive statistics from group 1 (ejaculated sperm subdivided according to the severity of sperm deficiency) and group 2 (testicular/epididymal sperm) including female age, number of mature oocytes (MII) retrieved following follicle aspiration, number of pre-embryos transferred to the uterine cavity. Values are mean $\pm S D$.

\begin{tabular}{lccccccc}
\hline & \multicolumn{3}{c}{$\begin{array}{c}\text { Group 1-Ejaculated Sperm } \\
(\mathrm{n}=220)\end{array}$} & \multicolumn{3}{c}{$\begin{array}{c}\text { Group 2-Testicular and Epididymal } \\
\text { Sperm from Azoospermic Men } \\
(\mathrm{n}=93)\end{array}$} \\
& $\begin{array}{l}\text { Normal } \\
(\mathrm{n}=86)\end{array}$ & $\begin{array}{c}\text { Single Defect } \\
(\mathrm{n}=41)\end{array}$ & $\begin{array}{c}\text { Double Defect } \\
(\mathrm{n}=45)\end{array}$ & $\begin{array}{c}\text { Triple Defect } \\
(\mathrm{n}=48)\end{array}$ & $\begin{array}{c}\text { OA } \\
(\mathrm{n}=39)\end{array}$ & $\begin{array}{c}\text { NOA } \\
(\mathrm{n}=54)\end{array}$ & p Value \\
\hline Female age (yrs.) & $34.7 \pm 4.7$ & $32.4 \pm 3.8$ & $31.6 \pm 5.6$ & $32.8 \pm 5.1$ & $32.6 \pm 7.4$ & $33.6 \pm 6.5$ & $\mathrm{NS}$ \\
$\begin{array}{l}\text { Mature oocytes } \\
\text { retrieved (n) }\end{array}$ & $8.6 \pm 5.9$ & $8.5 \pm 4.7$ & $9.3 \pm 6.2$ & $7.9 \pm 4.6$ & $9.6 \pm 5.5$ & $10.6 \pm 5.3$ & $\mathrm{NS}$ \\
Embryo transfer (n) & $3.3 \pm 1.4$ & $3.5 \pm 1.2$ & $3.6 \pm 1.3$ & $3.2 \pm 1.6$ & $3.4 \pm 1.3$ & $3.0 \pm 1.6$ & $\mathrm{NS}$ \\
\hline
\end{tabular}

One-way ANOVA was used to compare clinical and laboratorial parameters among groups; $p<0.05$ was considered significant; NS = not significant; $O A=$ obstructive azoospermia; NOA = non-obstructive azoospermia, values are mean \pm standard deviation.

Table 2 - ICSI outcomes with ejaculated sperm with and without sperm defects, and with epididymal and testicular spermatozoa from men with obstructive and non-obstructive azoospermia.

\begin{tabular}{lllllll} 
& \multicolumn{2}{c}{$\begin{array}{c}\text { Group 1 - Ejaculated Sperm } \\
(\mathrm{n}=220)\end{array}$} & \multicolumn{3}{c}{$\begin{array}{c}\text { Group 2 - Testicular and Epididymal Sperm } \\
\text { from Azoospermic Men }(\mathrm{n}=93)\end{array}$} \\
$\begin{array}{l}\text { Normal } \\
(\mathrm{n}=86)\end{array}$ & $\begin{array}{c}\text { Single Defect } \\
(\mathrm{n}=41)\end{array}$ & $\begin{array}{c}\text { Double Defect Triple Defect } \\
(\mathrm{n}=45)\end{array}$ & $\begin{array}{c}\text { OA } \\
(\mathrm{n}=48)\end{array}$ & $\begin{array}{c}\text { NOA } \\
(\mathrm{n}=39)\end{array}$ & p Value \\
$(\mathrm{n}=54)$ & \\
$71.3 \pm 24.1^{\mathrm{a}}$ & $73.2 \pm 22.1^{\mathrm{b}}$ & $72.1 \pm 19.6^{\mathrm{c}}$ & $63.4 \pm 26.9^{\mathrm{d}}$ & $73.6 \pm 20.7^{\mathrm{e}}$ & $52.2 \pm 29.3^{\mathrm{f}}$ & $<0.05^{\mathrm{d}, \mathrm{f} \text { versus a,b,c,e }}$ \\
$92.8 \pm 18.1^{\mathrm{a}}$ & $89.6 \pm 21.4^{\mathrm{b}}$ & $93.5 \pm 17.4^{\mathrm{c}}$ & $88.1 \pm 28.6^{\mathrm{d}}$ & $94.8 \pm 10.2^{\mathrm{e}}$ & $77.7 \pm 34.0^{\mathrm{f}}$ & $<0.05^{\mathrm{f} \text { versus a,b,c,d,e }}$ \\
& & & & & & \\
$48.4 \pm 34.8^{\mathrm{a}}$ & $50.5 \pm 30.9^{\mathrm{b}}$ & $46.9 \pm 31.1^{\mathrm{c}}$ & $48.3 \pm 35.8^{\mathrm{d}}$ & $46.3 \pm 30.0^{\mathrm{e}}$ & $35.7 \pm 27.4^{\mathrm{f}}$ & $<0.05^{\mathrm{f} \text { versus a,b,c,d,e }}$
\end{tabular}

Fertilization

$(\% 2 \mathrm{PN})$

Cleavage

$(\%)$

(1)

Good embryo

quality* $(\%)$

$48.4 \pm 34.8^{\mathrm{a}} \quad 50.5 \pm 30.9^{\mathrm{b}} \quad 46.9 \pm 31.1^{\mathrm{c}}$

$48.3 \pm 35.8-46.3 \pm 30.0^{\mathrm{c}}$

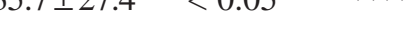

Clinical pregnancy

$40.9^{\mathrm{a}}$

$36.6^{\mathrm{b}}$

$44.4^{\mathrm{c}}$

$51.0^{\mathrm{d}}$

$51.3^{\mathrm{e}}$

$25.9^{\mathrm{f}}$

$0.01^{\mathrm{f} \text { versus a,b,c,d,e }}$

Miscarriage

$(\%)$

14.9

9.1

12.5

12.0

20.0

14.3

NS

One-way ANOVA was used to compare clinical and laboratorial parameters among groups, and the chi-square test was used to compare pregnancy and miscarriage rates, $p<0.05$ considered significant; $N S=$ not significant. $*=7-9$ blastomeres of similar size, and grades I or II cytoplasmic fragmentation on the day of embryo transfer (day 3) (17). OA = obstructive azoospermia; NOA = non-obstructive azoospermia, values are mean \pm standard deviation. 
Table 3 - ICSI outcomes using epididymal and testicular sperm retrieved from obstructive azoospermic men.

\begin{tabular}{lccc}
\hline & $\begin{array}{c}\text { Epididymal Sperm } \\
(\mathrm{n}=31)\end{array}$ & $\begin{array}{c}\text { Testicular Sperm } \\
(\mathrm{n}=08)\end{array}$ & p Value \\
\hline Female age (years) & $31.5 \pm 7.7$ & $36.3 \pm 5.1$ & $\mathrm{NS}$ \\
Mature oocytes retrieved (n) & $9.4 \pm 5.8$ & $9.4 \pm 4.9$ & $\mathrm{NS}$ \\
Embryo transfer (n) & $3.3 \pm 1.3$ & $3.7 \pm 1.5$ & $\mathrm{NS}$ \\
Fertilization $(\%$ 2PN) & $74.7 \pm 21.2$ & $69.1 \pm 19.6$ & $\mathrm{NS}$ \\
Cleavage $(\%)$ & $95.5 \pm 9.0$ & $92.2 \pm 14.4$ & $\mathrm{NS}$ \\
Good embryo quality $*(\%)$ & $44.6 \pm 30.5$ & $52.7 \pm 29.6$ & NS \\
Clinical pregnancy $(\%)$ & 51.6 & 50.0 & - \\
Miscarriage $(\%)$ & 18.8 & 25.0 & - \\
\hline
\end{tabular}

Fisher exact test was used to compare clinical and laboratorial parameters between groups. Clinical pregnancy and miscarriage rates were not compared due to the insufficient sample size. $P<0.05$ considered significant; $N S=$ not significant; * $=7-9$ blastomeres of similar size, and grades I or II cytoplasmic fragmentation on the day of embryo transfer (day 3) (17). OA: obstructive azoospermia, values are mean \pm standard deviation.

verity of sperm abnormalities found in the semen analysis. Most studies evaluating ICSI and azoospermia take into account only the sperm source but not the type of azoospermia. These studies tend to have a better outcome when epididymal spermatozoa are used, but these findings can be justified by the fact that epididymal sperm are always from obstructive azoospermia, while testicular sperm can be from both types of azoospermia $(20,22)$.

Apart from very few studies, there is no systematic differentiation between obstructive and nonobstructive azoospermia. This prevents consideration of the importance of spermatogenesis defects and of sperm immaturity on fertilization and embryo development. It is also true for ejaculated sperm in which spermatogenesis can vary greatly in a given ejaculate. Sperm samples can be within the normal ranges according to the WHO criteria, but can also harbor sperm with defects in count, motility and morphology. Therefore, it is reasonable to speculate that the fertilizing potential of these gametes may differ. In fact, ICSI outcomes with testicular spermatozoa from nonobstructive azoospermic men have been poorer than ejaculated and epididymal sperm $(2-4,21)$. In addition, miscarriage rates after ICSI with testicular spermatozoa have been reported to be higher $(5,7)$, although a clear distinction in the type of azoospermia is not always possible to analyze. These findings strengthen the importance of the type of azoospermia and not only the sperm source.

Our study took into consideration a different point of view from the previous studies by subdividing sperm deficiencies seen on semen analyses by degree of severity and also by the type of azoospermia. We observed lower fertilization rates when ejaculated spermatozoa from oligoasthenoteratozoospermic (triple defect) men were used for ICSI as compared to the other ejaculated samples, and also in relation to testicular or epididymis spermatozoa from the obstructed azoospermic men. From all subgroups, testicular spermatozoa from NOA patients had the worst performance after ICSI. We observed significantly lower fertilization and embryo development as well as pregnancy rates when compared to the other subgroups. However, our study was unable to identify differences in miscarriage among groups as shown by others $(5,7)$. Although there is a tendency for higher miscarriage rates in our group of azoospermic patients, the sample size is limited to allow proper analysis.

Our results indicate that sperm from men with severely altered spermatogenesis, such as ejaculated sperm in OAT and testicular sperm in NOA, have decreased fertility potential after ICSI. We speculate that in terms of severity, non-obstructive azoospermia (NOA) may be a progression of oligoasthenoteratozoospermia (OAT), thus justifying the diminished per- 
formance of sperm from both conditions in ICSI. Differently, in OA cases the absence of sperm in the ejaculate is exclusively due to an obstruction in some point of the ductal system, but the spermatogenesis is normal. ICSI results in this condition is independent of the sperm source, i.e., sperm retrieved from the epididymis or testicles perform similarly and at the same extent of normal to mild/moderate abnormal (single or double defects) ejaculated spermatozoa. Besides, we observed that normal fertilization rates with OA sperm was significantly higher when compared to sperm obtained from OAT ejaculated semen, thus reinforcing the hypothesis that the spermatic defect severity is more important than the sperm source in ICSI results.

Indeed, sperm from men with severely defective spermatogenesis may have a higher tendency to carry deficiencies, such as the ones related to the centrioles and genetic material, which ultimately affects the capability of the male gamete to activate the egg and trigger the formation and development of a normal zygote and a viable pre-embryo. A higher chromosomal aneuploidy rate and other genetic alterations $(9,23)$ have been found in spermatozoa from NOA and OAT men. Pang et al. (24) in studies of sexual chromosomes and other 12 autosomes showed a higher incidence of chromosome aneuploidy rate in sperm of men with OAT versus fertile controls, varying from $33-74 \%$ in the first group versus $4.1-7.7 \%$ in the control. Recent studies (25) tend to differentiate the contribution of the male gamete (termed 'paternal effect') to early and late embryo development. Lower fertilization rates, as observed in our study for OAT sperm and for testicular sperm from NOA men, are explained by early paternal effects, which include alterations in spermatic cytosolic factor and are responsible for the completion of the oocyte meiotic division as well as alterations on sperm centriole, which participate in the formation of embryo mitotic fuses in early cellular divisions (25). Other alterations such as sperm DNA fragmentation would be associated to the so called late paternal effect that impacts embryo implantation. Recent studies show lower pregnancy rates when the proportion of sperm with fragmented DNA in a given sample is above $10 \%$ and absence of pregnancy when the DNA fragmentation is above $25 \%$ (26).

\section{CONCLUSION}

Adequate fertilization, cleavage and pregnancy rates are to be expected when ICSI is performed with ejaculated spermatozoa from men with mild to moderate sperm alterations or from azoospermic men with normal sperm production, such as the obstructive azoospermic ones. However, lower fertilization rates are achieved when ICSI is performed with sperm from men with oligoasthenoteratozoospermia and non-obstructive azoospermia. Also, embryo development and pregnancy rates are significantly lower when ICSI is used with testicular spermatozoa from NOA men. Although ICSI is a formidable therapy that trespasses obstacles faced by sperm in its function as a carrier, it cannot alter the message carried by the male gamete.

\section{CONFLICT OF INTEREST}

None declared.

\section{REFERENCES}

1. Palermo G, Joris H, Devroey P, Van Steirteghem AC: Pregnancies after intracytoplasmic injection of single spermatozoon into an oocyte. Lancet. 1992; 340: 17-8.

2. Friedler S, Raziel A, Strassburger D, Schachter M, Soffer Y, Ron-El R: Factors influencing the outcome of ICSI in patients with obstructive and non-obstructive azoospermia: a comparative study. Hum Reprod. 2002; 17:3114-21.

3. De Croo I, Van der Elst J, Everaert K, De Sutter P, Dhont M: Fertilization, pregnancy and embryo implantation rates after ICSI in cases of obstructive and non-obstructive azoospermia. Hum Reprod. 2000; 15: 1383-8.

4. Nicopoullos JD, Gilling-Smith C, Almeida PA, Ramsay JW: The results of 154 ICSI cycles using surgically retrieved sperm from azoospermic men. Hum Reprod. 2004; 19: 579-85.

5. Pasqualotto FF, Rossi LM, Guilherme P, Ortiz V, Iaconelli A Jr, Borges E Jr: Etiology-specific outcomes of intracytoplasmic sperm injection in azoospermic patients. Fertil Steril. 2005; 83: 606-11.

6. Vernaeve V, Tournaye H, Osmanagaoglu K, Verheyen G, Van Steirteghem A, Devroey P: Intracytoplasmic 
sperm injection with testicular spermatozoa is less successful in men with nonobstructive azoospermia than in men with obstructive azoospermia. Fertil Steril. 2003; 79: 529-33.

7. Buffat C, Patrat C, Merlet F, Guibert J, Epelboin S, Thiounn N: ICSI outcomes in obstructive azoospermia: influence of the origin of surgically retrieved spermatozoa and the cause of obstruction. Hum Reprod. 2006; 21: 1018-24.

8. Balaban B, Urman B, Isiklar A, Alatas C, Mercan R, Aksoy S: Blastocyst transfer following intracytoplasmic injection of ejaculated, epididymal or testicular spermatozoa. Hum Reprod. 2001; 16: 125-9.

9. Mateizel I, Verheyen G, Van Assche E, Tournaye H, Liebaers I, Van Steirteghem A: FISH analysis of chromosome $\mathrm{X}, \mathrm{Y}$ and 18 abnormalities in testicular sperm from azoospermic patients. Hum Reprod. 2002; 17: 2249-57.

10. World Health Organization: Laboratory Manual for the Examination of Human Semen and Sperm-Cervical Mucus Interaction. 4th edition. Cambridge: Cambridge University Press, 1999.

11. Kruger TF, Menkveld R, Stander FS, Lombard CJ, Van der Merwe JP, van Zyl JA, et al.: Sperm morphologic features as a prognostic factor in in vitro fertilization. Fertil Steril. 1986; 46: 1118-23.

12. Gunalp S, Onculoglu C, Gurgan T, Kruger TF, Lombard CJ: A study of semen parameters with emphasis on sperm morphology in a fertile population: an attempt to develop clinical thresholds. Hum Reprod. 2001; 16: $110-4$.

13. Ombelet W, Bosmans E, Janssen M, Cox A, Vlasselaer $\mathrm{J}$, Gyselaers W, et al.: Semen parameters in a fertile versus subfertile population: a need for change in the interpretation of semen testing. Hum Reprod. 1997; 12: 987-93.

14. Lundin K, Soderlund B, Hamberger L: The relationship between sperm morphology and rates of fertilization, pregnancy and spontaneous abortion in an in-vitro fertilization/intracytoplasmic sperm injection programme. Hum Reprod. 1997; 12: 2676-81.

15. Coetzee K, Kruge TF, Lombard CJ: Predictive value of normal sperm morphology: a structured literature review. Hum Reprod Update. 1998; 4: 73-82.

16. Schlegel PN, Palermo GD, Goldstein M, Menendez S, Zaninovic N, Veeck LL, et al.: Testicular sperm extraction with intracytoplasmic sperm injection for nonobstructive azoospermia. Urology. 1997; 49: 435-40.

17. Veeck LL: Preembryo Grading. In: Veeck LL (ed.), Atlas of the human oocyte and early conceptus. Baltimore, Williams \& Wilkins. 1999; Vol. 2, pp. 62-144.
18. Glantz SA: Primer of Bio-Statistics. 4th Edition. New York, 1997; pp. 323-99.

19. Nicopoullos JD, Gilling-Smith C, Ramsay JW: Does the cause of obstructive azoospermia affect the outcome of intracytoplasmic sperm injection: a meta-analysis. BJU Int. 2004; 93: 1282-6.

20. Ghazzawi IM, Sarraf MG, Taher MR, Khalifa FA: Comparison of the fertilizing capability of spermatozoa from ejaculates, epididymal aspirates and testicular biopsies using intracytoplasmic sperm injection. Hum Reprod. 1998; 13: 348-52.

21. Mansour RT, Kamal A, Fahmy I, Tawab N, Serour GI, Aboulghar MA: Intracytoplasmic sperm injection in obstructive and non-obstructive azoospermia. Hum Reprod. 1997; 12: 1974-9.

22. Goker EN, Sendag F, Levi R, Sendag H, Tavmergen E: Comparison of the ICSI outcome of ejaculated sperm with normal, abnormal parameters and testicular sperm. Eur J Obstet Gynecol Reprod Biol. 2002; 104: 129-36.

23. Liu CH, Tsao HM, Cheng TC, Wu HM, Huang CC, Chen CI, et al.: DNA fragmentation, mitochondrial dysfunction and chromosomal aneuploidy in the spermatozoa of oligoasthenoteratozoospermic males. J Assist Reprod Genet. 2004; 21: 119-26.

24. Pang MG, Hoegerman SF, Cuticchia AJ, Moon SY, Doncel GF, Acosta AA, et al.: Detection of aneuploidy for chromosomes 4, 6, 7, 8, 9, 10,11, 12, 13, 17, 18, 21, X and $\mathrm{Y}$ by fluorescence in-situ hybridization in spermatozoa from nine patients with oligoasthenoteratozoospermia undergoing intracytoplasmic sperm injection. Hum Reprod. 1999; 14: 1266-73.

25. Tesarik J: Paternal effects on cell division in the human preimplantation embryo. Reprod Biomed Online. 2005; 10:370-5.

26. Henkel R, Kierspel E, Hajimohammad M, Stalf T, Hoogendijk C, Mehnert C, et al.: DNA fragmentation of spermatozoa and assisted reproduction technology. Reprod Biomed Online. 2003; 7: 477-84.

Accepted after revision:

October 23, 2006

\section{Correspondence address:}

Dr. Sandro Esteves

Av. Dr. Heitor Penteado, 1464

Campinas, SP, 13075-460, Brazil

Fax: + 55 193294-6992

E-mail: s.esteves@ androfert.com.br 